ESAIM: PROCEEDINGS AND SURVEYS, December 2016, Vol. 55

Emmanuel FRÉNOD, Emmanuel MAITRE, Antoine ROUSSEAU, Stéphanie SALMON and Marcela SZOPOS Editors

\title{
FOREWORD TO THE PROCEEDINGS OF CEMRACS 2015
}

\author{
Emmanuel Frénod ${ }^{1}$, Emmanuel Maitre ${ }^{2}$, Antoine Rousseau ${ }^{3}$, Stéphanie \\ SALMON $^{4}$ AND MARCELA SzOPOS ${ }^{5}$
}

Abstract. 20th CEMRACS Summer School : contributions

CEMRACS (Summer Mathematical Research Center on Scientific Computing and Applications) is a 6-week scientific event that is organized every year at CIRM, a CNRS mathematical institute located in the Calanques National Park near the city of Marseille, France. Under the auspices of the SMAI, its goal is to gather academic and industrial researchers to get an accurate focus on scientific computing problems that are relevant to both communities.

This event is shared into two periods : A one weak summer school followed by a five weeks research session.

CEMRACS 2015 was the twentieth of the series. It was devoted to the simulation of (coupled) multi-physics models involving fluids.

The summer school focused on fluid coupling in life sciences; with the following lectures:

- Igor Aronson (Argonne National Lab, Chicago): Collective behavior in active systems.

- Didier Bresch (CNRS - LAMA, Université de Savoie): Hierarchy of fluid models and environmental problem.

- Alberto Figueroa (College of Engineering and Medical School, University of Michigan): Image-based modeling of the cardiovascular system.

- Céline Grandmont (Inria - LJLL, Université Pierre et Marie Curie): Fluid-Structure interaction.

- Paul Vigneaux (ENS Lyon - UMPA): Small scale fluid dynamics, interfaces and numerics.

One aim of the project session was to stimulate exchanges between faculty members (applied mathematicians, geophysicists or biologists) and industrial partners.

The articles presented in this volume are the result of these research session, which gathered an average of 35 researchers every week (for a total of about 100 participants for the whole research session) working on 9 projects. Those projects focussed on the numerical methods and their implementation for

- Intestinal mucus modelling;

- Crowd movement;

- Cell displacement;

- Cerebral angiography

\footnotetext{
1 Université Bretagne Sud - LMBA

2 Université de Grenoble - LJK

${ }^{3}$ Inria Montpellier - Lemon

${ }^{4}$ Université de Reims Champagne - Ardennes

5 Université de Strasbourg - Irma
} 
- Physic based preconditioning for discontinuous Galerkin Shallow Water scheme;

- Simulation of a bioreactor;

- Depressurization process at low Mach number in nuclear reactor;

- Geometric Multigrid for Box-Splines;

- Coupled Exner-Stokes equation.

The sponsors of CEMRACS 2015, who allowed the researcher's facilities were ANR, CNRS, Inria, Smai, Alsace Biovalley, LyonBioPole, Meso Centre of Marseille, Max-Planck IPP (Garching, Germany), transfer structures MaiMoSiNE and Cemosis and Labex Amies, Centre Henri Lebesgue, Numev and Irmia.

We address our warmest thanks to the whole team at CIRM for their skills and spirit that allowed us to work during these research sessions in such a good mood, to the colleagues from SMAI for their kind and efficient help on the technical matters (Emmanuel Gobet, Pauline Lafitte, Alain Prignet and Noura Sahtout), to Alexandre Ancel for his valuable expertise on the scientific computing issues and Annie Aliaga for her action on administrative questions.

We finally address also our thanks to the participants of the summer school and the research projects, who happily spent part of their summer to make progresses on these useful problems. 